\title{
Closed Form Metrics to Accurately Model the Response in General Arbitrarily-Coupled $R C$ Trees
}

\author{
Dinesh Pamunuwa* and Shauki Elassaad ${ }^{* *}$ \\ *Laboratory of Electronics and Computer Systems, Dept. of Microelectronics and Information Technology \\ Royal Institute of Technology, Stockholm, Sweden.e-mail: dinesh@imit.kth.se \\ **Cadence Berkeley Laboratories, Cadence Design Systems, Berkeley, CA.e-mail: shauki@cadence.com
}

\begin{abstract}
Closed form expressions are presented for the first and second moment of the impulse response for arbitrarily-coupled $R C$ trees with multiple drivers, and used to generate accurate second order estimations of the transfer function from any driver to the receiver. The superposition of the waveforms for all switching events allows precise delay and noise calculations for systems of coupled interconnects with different aggressor arrival times, with a minimum of computational complexity.
\end{abstract}

\section{INTRODUCTION}

As gate transition times decrease and wiring density increases, particular attention has to be paid to noise modeling and its impact on performance and functionality. Delay models that account for the coupling noise induced by a number of switching neighboring aggressors on a victim signal are necessary. Finding the response of such systems requires the analysis of general arbitrarily-coupled trees. However the computational overhead associated with numeric techniques such as Spice is usually unacceptable for large designs. This has led to a proliferation of research in the past twenty years in the area of delay and noise modeling, with the aim of developing simplified metrics that still give acceptable accuracy. In the rest of this document, the term simple tree is used to refer to a tree that has capacitances only to ground, and coupled tree to refer to a tree that consists of simple trees coupled to each other through series capacitors.

There is a large body of literature that deals with delay modeling in simple trees. One of the most important and widely used metrics, the first moment of the impulse response, was proposed back in 1948 as an upper bound for the delay in valve circuits [1], and is known as the Elmore delay. Subsequently the authors of [2] developed tighter bounds and metrics that gave an indication of when the Elmore model was poor. Its attraction is that it is very simple, and yet exhibits good fidelity, giving results as good as more expensive models when used as a metric in interconnect optimization algorithms. A model based on the first and second moment of the impulse response, and the sum of the open circuit time constants was proposed in [3], which gives a stable approximation to the second order transfer function for simple trees. Since then, generic moment based techniques have been developed which are applicable to any linear circuit, and allow the calculation of an arbitrary number of poles [4]. In today's systems, delay and noise calculations are essential at an early stage in the planning process. However the complexity is such that generic moment matching and model order reduction techniques which require the formulation of nodal matrices and costly matrix manipulations are too expensive. Hence a lot of simplified models have been proposed. The models of [3] represent the minimum complexity for second order approximations of simple trees. In [5] the authors explicitly match the first three moments of the impulse response to a second order model in a methodology that guarantees stability. In [6] a heuristic delay model based on the first two moments was proposed.

In analyzing coupled trees, most research has concentrated on certain simplified configurations of interest. In [7] the authors present two pole delay models for a single $\pi$ section, and extend it to accommodate multiple segmented aggressors in [8], but the allowed topology is still limited. In [9] the authors use circuit transformations to simplify a general tree to a $2-\pi$ model when analytic formulae can be used, but intermediate steps require the calculation of admittances at each branch point and the estimation of equivalent capacitances which increase run time and impact on the accuracy respectively. In [10] a technique is presented to generate the poles of a system with $n$ storage elements, which has long been used in analog design to estimate the bandwidth of amplifiers. The complexity of the computation is proportional to $n^{\alpha}$ where $\alpha$ is the order of the pole. There are works which use this technique to estimate the two lowest frequency poles and use them to model the response for all switching events on the tree [11][12]. This can result in unacceptable accuracy, as the poles which determine the response for different switching events can be very far apart on the frequency axis. If used to generate higher frequency poles, this estimation technique will become prohibitively expensive.

In this paper we propose metrics based on the first and second moment of the impulse response to generate second order transfer functions from each driver to the receiver in arbitrarily-coupled trees, which allow both delay and noise estimations. Our contributions are that the moments are matched to the characteristic time constants in a novel way which minimizes computational complexity while allowing good accuracy, and that the moments themselves are calculated from completely accurate closed form expressions which are only slightly more complex than the Elmore delay, while retaining all its elegance. In this paper we are only concerned with the generation of the transfer function, which is the most important aspect of the modeling.

\section{MODELING}

The modeling requires the matching of easily calculable metrics of the circuit to the system transfer function. This involves 


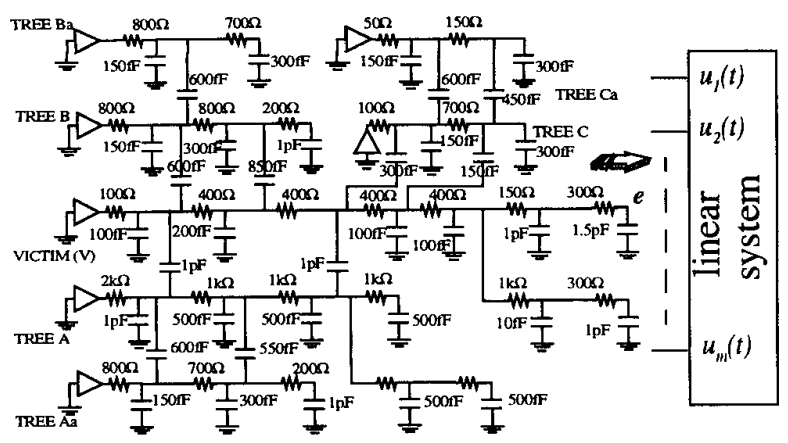

Figure 1. Example coupled $R C$ tree

ascertaining the characteristics of the transfer function, calculating the metrics and then matching them to the terms in the transfer function.

A coupled $R C$ tree is characterized by a resistive path from the receiver node $e$ to the forcing (victim) driver, and series capacitive elements to other (aggressor) drivers. Hence when the victim driver switches the output will always change rails, while it will start and end at the same rail for an aggressor switching. Therefore the transfer functions characterizing the response to the victim switching and any of the aggressors switching are different. The former will have a zero on the negative part of the real axis:

$$
H_{v}(s)=\frac{1+s \tau_{z, v}}{\left(1+s \tau_{1}\right)\left(1+s \tau_{2}\right)}
$$

while the latter will have a zero at the origin.

$$
H_{a_{i}}(s)=\frac{s \tau_{z, a_{i}}}{\left(1+s \tau_{1}\right)\left(1+s \tau_{2}\right)}
$$

\subsection{Calculation of moments}

In the following section, expressions are presented for the first and second moment of the impulse response for general coupled trees, which form the core of our models. The derivation is based on Kirchoff's laws and integration by parts, and is omitted due to lack of space. Shown in Fig. 1 is an example of a coupled tree which can be referred to in the following descriptions. First our notation is described below.

$C S_{k}^{p}=$ capacitance to ground at node $\mathrm{k}$ in pth tree

$C C_{k j}^{p q}=$ capacitance between node $\mathrm{k}$ on pth tree and node $\mathrm{j}$ on qth i tree (first sub(super)script refers to reference tree)

$R_{k e}^{p}=$ resistance shared on paths between source to nodes $\mathrm{e}$ and $\mathrm{k}$ on tree $\mathrm{p}$

$\mathrm{r}_{k}^{n}=\mathrm{nth}$ moment of the impulse response at the kth node

It should be noted that superscripts always refer to simple trees while subscripts always refer to nodes, except in the definition for moments, where the superscript refers to the order of the moment. Additionally, rail voltages are normalized to 0 and 1 , and the expressions always derived for a positive step without loss of generality.

The first moment of the impulse response at the receiver node $e$ for the victim driver switching is given by:

$$
\Upsilon_{e, v}^{1}=\int_{0}^{\infty} t h_{e}^{\nu}(t) d t
$$

Now the impulse response is the first time derivative of the step response, for which an expression can be formulated by summing up all capacitor currents. This can then be integrated by parts to yield (4), where $a_{1}, a_{2}$.. are the aggressors.

$$
\Upsilon_{e, v}^{\mathrm{l}}=\sum_{k \in \text { victim }} R_{k e}^{v}\left[C S_{k}^{\nu}+C C_{k}^{v a_{1}}+C C_{k}^{v a_{2}}+\ldots\right]=\tau_{D_{e}}^{v} \text { say }
$$

The second moment of the impulse response at $e$ is given by:

$$
\Upsilon_{e, v}^{2}=\int_{0}^{\infty} t^{2} h_{e}^{v}(t) d t
$$

Following the procedure described above in two stages, this can be shown to be equivalent to (6).

$$
\begin{aligned}
& \mathrm{r}_{e, v}^{2}=2 \sum_{k \in v i c t i m} R_{k e}^{v}\left\{C S_{k}^{v} \tau_{D_{k}}^{v}+C C_{k}^{v a_{1}}\left[\tau_{D_{k}}^{v}+\sum_{K \in a_{1}} R_{K j}^{a_{1}} C C_{K}^{a_{1} v}\right]\right. \\
& \left.+C C_{k}^{v a_{2}}\left[\tau_{D_{k}}^{v}+\sum_{K \in a_{2}} R_{K j}^{a_{2}} C C_{K}^{a_{2} v}\right]+\ldots\right\}=2\left(\tau_{G_{e}}^{v}\right)^{2} \text { say }
\end{aligned}
$$

From an approach identical to the former case, the first moment of the impulse response at node $e$ on the victim tree for aggressor $a_{i}$ switching can be shown to be:

$$
\Upsilon_{e, a_{i}}^{1}=-\sum_{k \in \text { victim }} R_{k e}^{v} C C_{k}^{v a_{i}}=-\tau_{D_{e}}^{a_{i}} \text { say }
$$

and the second moment:

$$
\begin{aligned}
& \Upsilon_{e, a_{i}}^{2}=-2 \sum_{k \in v i c t i m} R_{k e}^{v}\left\{\left(C S_{k}^{v}+C C_{k}^{v a_{1}}+C C_{k}^{v a_{2}}+\ldots\right) \tau_{D_{k}}^{a_{i}}\right. \\
& \left.+C C_{k}^{v a_{i}}\left[\sum_{K \in a_{i}} R_{K j}^{a_{i}}\left(C S_{K}^{a_{i}}+C C_{K}^{a_{i} v}+C C_{K}^{a_{i} b_{1}}+\ldots\right)\right]\right\} \\
& =-2\left(\tau_{G_{e}}^{a_{i}}\right)^{2} \text { say }
\end{aligned}
$$

The expressions in (4), (6), (7) and (8) form the basis of our proposed models. An examination of these reveal their similarity to the Elmore delay, and all the accompanying characteristics that make the estimation algorithms very efficient.

\subsection{Matching moments to the characteristic time constants in the circuit}

The moments can be matched to the characteristic time constants in the circuit by using the identity that the $n^{\text {th }}$ moment of the impulse response is $(-1)^{n}$ times the $n^{\text {th }}$ derivative of the transfer function evaluated at $s=0$. This identity used on (1), (4) and (6) results in:

$$
\begin{gathered}
\tau_{D_{e}}^{\nu}=\tau_{1}+\tau_{2}-\tau_{z, \nu} \\
\left(\tau_{G_{e}}^{\nu}\right)^{2}=\left(\tau_{1}+\tau_{2}-\tau_{z, \nu}\right)\left(\tau_{1}+\tau_{2}\right)-\tau_{1} \tau_{2}
\end{gathered}
$$


Now additional information is necessary to solve for the three unknowns in (9) and (10). If the reciprocal pole sum is designated as $\tau_{\text {sum }}$, these two equations can be combined to form the following quadratic, which yields two time constants.

$$
\tau^{2}-\tau_{\text {sum }} \tau+\tau_{D_{e}}^{\nu} \tau_{\text {sum }}-\left(\tau_{G_{e}}^{\nu}\right)^{2}=0
$$

The mathematical basis of our reduced order model is that geometric attributes (area and first moment of the area) and the values at $t=0$ and $t \rightarrow \infty$ of the estimated and actual waveform are equated. Since the initial and final values are already considered in the formulation of the transfer function, additional information is necessary to solve for the two poles and zeros associated with a switching event. At this point, it is helpful to look at the physical interpretation of the first and second moments of the impulse response. The first moment always considers resistances of the switching line, and either all capacitances connected to the switching line (in the case of the victim driver switching) or capacitances connecting it to a particular line (for the switching of an aggressor driver). The second moment propagates outwards another level, and considers the resistances and capacitances of immediately adjacent lines as well. This intuition is valuable in generating a solution with minimum computational complexity; namely, equation (11) can be used to generate the pole time constants for all switching events, by using the appropriate reciprocal pole sum.

For the victim switching, the metric that gives the best solution is the sum of the open circuit time constants with reference to the victim driver, which we shall call $\tau_{p}^{*}$. This is simply the summation of the products of all capacitances connected to the victim line with the driving point resistance to each of those capacitors. This is a good approximation for the sum of the pole time constants[10], giving:

$$
\tau_{p}^{*}=\tau_{1}+\tau_{2}
$$

To solve for the poles and zeros associated with an aggressor switching, the above identity is used on (2), (7) and (8) to give:

$$
\begin{gathered}
\tau_{D_{e}}^{a_{i}}=\tau_{z, a_{i}} \\
\left(\tau_{G_{e}}^{a_{i}}\right)^{2}=\tau_{z, a_{i}}\left(\tau_{1}+\tau_{2}\right)
\end{gathered}
$$

Now the zero time constant is available immediately, and dividing (14) by (13) results in the reciprocal pole sum:

$$
\left(\tau_{G}^{a_{i}}\right)^{2} / \tau_{D_{e}}^{a_{i}}=\tau_{,} \tau_{1}+\tau_{2}
$$

The pole time constants can be obtained by substituting (15) as $\tau_{\text {sum }}$ in (11). Since minimum information is used for both cases, some slight modification is necessary to guarantee stability, as explained below.

The conditions for potential instability can be identified by analyzing the quadratic which yields the time constants. The first limiting condition is that the magnitude of the square root should be greater than the reciprocal pole sum, which yields:

$$
\tau_{s u m}>\left(\tau_{G_{e}}^{\nu}\right)^{2} / \tau_{D_{e}}^{\nu}
$$

The second is that the sign under the radical is negative, which,
Table 1. Different values for $\tau_{\text {sum }}$ for an aggressor switching

\begin{tabular}{|c|c|}
\hline Condition & $\tau_{\text {sumi }}$ \\
\hline \hline no violation & $\left(\begin{array}{c}a_{i} \\
\left.\tau_{G}\right)^{2} / \tau_{D_{e}}\end{array}\right.$ \\
\hline$\left(\begin{array}{c}\tau_{i} \\
\left.{ }^{G_{e}}\right)^{2} / \tau_{D_{e}}{ }^{a_{i}}<\left(\tau_{G}^{v}\right)^{2} / \tau_{D_{e}}^{v}\end{array}\right.$ & $0.99\left(\tau_{G}^{v}\right)_{e}^{2} / \tau_{D_{e}}^{v}$ \\
& $0.02\left[\tau_{D_{e}}^{v}-\sqrt{\left(\tau_{D_{e}}^{v}\right)^{2}-\left(\tau_{G}^{v}\right)^{2}}\right]$ \\
\hline
\end{tabular}

after some simplification, results in the following:

$$
\begin{gathered}
\tau_{s u m}<2\left[\tau_{D_{e}}^{v}-\sqrt{\left(\tau_{D_{e}}^{v}\right)^{2}-\left(\tau_{G_{e}}^{v}\right)^{2}}\right] \text { or } \\
\tau_{s u m}>2\left[\tau_{D_{e}}^{v}+\sqrt{\left(\tau_{D_{e}}^{v}\right)^{2}-\left(\tau_{G_{e}}^{v}\right)^{2}}\right]
\end{gathered}
$$

An inspection of the relevant expressions shows that the only possible violation in the case of the victim driver switching is (B). i.e. very occasionally, using $\tau_{p}^{*}$ can result in complex poles. The physical interpretation of such an occurrence is that the sum of the open circuit time constants underestimates the reciprocal pole sum, which has been unusually escalated by an aggressor or aggressors with exceptionally high parasitics. Because both exponential waveforms are either additive or subtractive unlike when an aggressor switches (where one is additive and the other is subtractive), the higher frequency pole does not have a significant impact. In fact, this form of instability is usually an indication of a very low frequency pole which makes the prediction of the waveform straightforward. The simplest remedy therefore is to consider a single pole response, with the pole time constant being given by $\tau_{D_{e}}^{v}$, which results in good accuracy as shown in the results section.

For the case of an aggressor driver switching, the only possible violation is (A). This is in fact the more common form of instability encountered and occurs when the dominant poles for the victim and the particular aggressor are very far apart on the frequency axis. Physically, this translates to a situation where the receiver node is charged extremely rapidly by a very strong aggressor (i.e. through a relatively very small time constant), and decays with a very long tail, dictated by the much larger time constant of the victim. Such behavior is common for far end coupling, The instability in the solution predicted by (11) occurs because the reciprocal pole sum given by (15) accurately reflects the high frequency nature of the poles in the aggressor's charging path, but $\tau_{D_{e}}^{v}$ and $\left(\tau_{G}^{v}\right)^{2}$ reflect the much lower frequency content of the victim's dominant poles, and the gap is too much to bridge. The solution without generating extra information about the circuit, is to accept the next best approximation. That is to say, if $\tau_{\text {sum }}$ is so small that it violates inequality (A), the simplest and most logical remedy is to increase $\tau_{\text {sum }}$ so that real roots are generated. Since the equality will generate coincident poles which is not acceptable, the exact value should be chosen so that it is slightly greater than the equality, which can be achieved with a percentage factor, such as $1 \%$. This yields accurate results, because the intention is to generate the best two pole single zero 


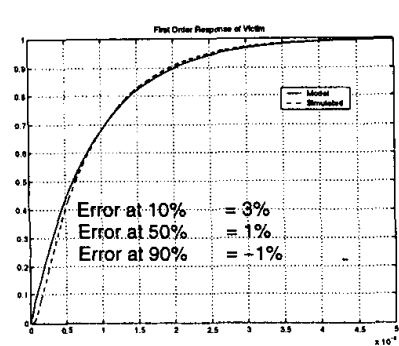

Fig.5 a Victim Switching

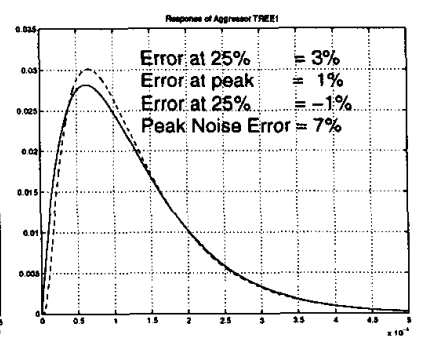

Fig.5.b Tree B Switching

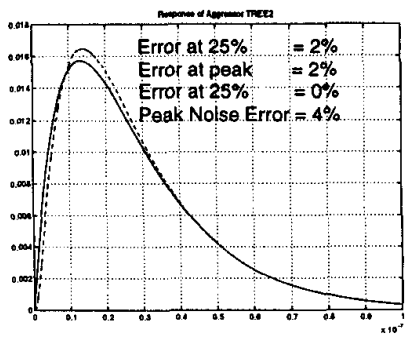

Fig.5.c Tree A Switching

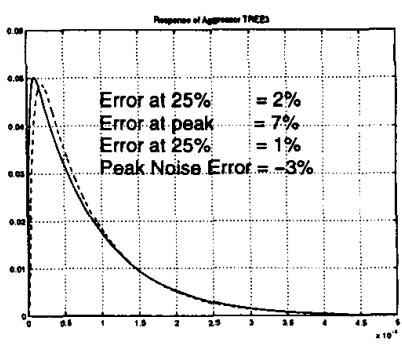

Fig.5.d Tree C Switching

Figure 2. Response to different switching events

model; in other words the poles and zero need not equate to actual poles and zeros of the system, and indeed should differ for a second order approximation. Using the factor of $1 \%$ beyond the threshold which yields coincident poles ensures that both the high and low frequency behavior is matched. Following this approach, the values that $\tau_{\text {sum }}$ should take in the different cases are summarized in Table 1.

\section{RESULTS}

The proposed metrics were tested on several different test beds which cover a wide range of topologies and all the corner cases where conditions (A) and (B) are violated, by comparing the step response for different switching events with Spectre simulations. Due to space restrictions, only the results pertaining to the tree shown in Fig. 1 consisting of the victim, three primary aggressors, and three secondary aggressors are presented. Shown in Fig. 2 are the waveforms (step response) at the receiver node $e$ for each driver switching. It can be seen that the model prediction is very close to the Spectre simulation. In the case of the victim switching, inequality (A) is violated, and the single pole response shows good accuracy. Since the actual and predicted delay at a single threshold can agree very well, and still result in significant deviations along the full waveform, we tested the accuracy at three points along the waveform. For the victim switching, the thresholds are $10 \%, 50 \%$ and $90 \%$, while for the aggressors they are $25 \%, 50 \%$ and $75 \%$ of the peak amplitude. The error at different thresholds is given as a fraction of the pulse width from $v_{1}$ to $v_{3}$ for the aggressors.

\section{CONCLUSIONS}

Closed form expressions for the first two moments of the impulse response for general coupled $R C$ trees were presented, and used to generate second order approximations to the transfer function for any switching event with guaranteed stability. The summation of all waveforms results in the complete response at the node of interest. The new models we propose represent the minimum complexity for second order estimations of coupled trees when generality is not compromised, and in fact subsume a lot of models that address simplified structures. For testing purposes, these expressions were used to derive the time domain waveform for the step response. The accuracy with which the delay at a given threshold is predicted was found to be more than
$90 \%$, even for complex circuits such as shown in Fig. 1. The peak noise was predicted with similar accuracy on circuits representative of nano meter interconnect structures. The simplicity and accuracy of the models combined with their generality should make them useful in delay and noise estimations in complex systems, early in the design flow.

\section{REFERENCES}

[1] W. C. Elmore, "The transient response of linear damped circuits," J. Appl. Physics, vol. 19, pp-55-63, Jan. 1948.

[2] J. Rubinstein, P. Penfield, and M. Horowitz, "Signal delay in RC tree networks," IEEE Trans. Computer-Aided Design, vol CAD-2, no. 3, pp. 202-211, Jul. 1983.

[3] M. A. Horowitz, "Timing models for MOS circuits," Ph.D. dissertation, Stanford Electronics Laboratories, Stanford University, Stanford, CA, Jan. 1984.

[4] L. T. Pillage and R. A. Rohrer, "Asymptotic waveform evaluation for timing analysis," IEEE Trans. Computer-Aided Design of ICs and Sys., vol. 9, pp. 352-366, Apr. 1990.

[5] B. Tutuianu, F. Dartu and L. T. Pillage, "An explicit RC-circuit delay approximation based on the first three moments of the impulse response," in Proc. DAC, 1996, pp. 611-616.

[6] C. J. Alpert, A. Devgan, and C. V. Kashyap, "RC Delay metrics for performance optimization," IEEE Trans. ComputerAided Design of ICs and Sys., vol. 20, no. 5, pp. 571-582. May 2001.

[7] A. B. Kahng, S. Muddu, and D. Vidhani, "Noise and delay uncertainty studies for coupled RC interconnects," in Proc. ASIC/SOC, 1999, pp. 3-8.

[8] A. B. Kahng, S. Muddu, N. Pol, and D. Vidhani, "Noise model for multiple segmented coupled RC interconnects," in Proc. ISQED, 2001, pp. 145-150.

[9] M. Takahashi, M. Hashimoto, and H. Onodera, "Crosstalk noise estimation for generic RC trees", in Proc. ICCD, 2001, pp. 110-116.

[10]B. L. Cochrun and A. Grabel, "On the determination of the transfer function of electronic circuits," IEEE Trans. Circuit Theory, vol. CT-20, pp.16-20, Jan. 1973.

[11]X. Tong and M. Marek-Sadowska, "Efficient delay calculation in presence of crosstalk," in Proc. ISQED, 2000, pp. 491-497.

[12]L. H. Chen and M. Marek-Sadowska, "Efficient closed-form cross-talk delay metrics," in Proc. ISQED, 2002, pp. 431436. 